\title{
Correlación entre la variabilidad en la banda VLF en Francisco Morazán y la actividad solar
}

Adán Artola, Edward Milla, Paolo Estrada

\section{Resumen}

En este artículo se analiza la correlación entre las fulguraciones solares y las Perturbaciones Súbitas en la lonosfera terrestre (SID) registradas utilizando la variabilidad en la intensidad de las ondas de radio de Muy Baja Frecuencia (VLF) reflejadas y refractadas en la capa $D$ de la ionosfera y monitoreadas en Francisco Morazán en el mes de febrero de 2014.

Para este fin se construyeron dos sistemas de monitoreo con configuraciones distintas las cuales aseguren una mejor calidad en el registro de los datos. El primer sistema utiliza el monitor VLF desarrollado por la Asociación de Radioastronomía del Reino Unido (UKRAA), que trabaja con un ancho de banda de 12 a $35 \mathrm{kHz}$, con una salida en de 0-5 volts que representa las variaciones en la frecuencia de 24 $\mathrm{kHz}$ transmitida por NAA en Cutler, Maine. El otro sistema aprovecha las ventajas de la Radio Definida a través de Software (SDR), el cual sin la necesidad de contar con la electrónica compleja de un receptor como el UKRAA, puede interpretar los cambios de energía detectados por la antena utilizando una tarjeta de sonido como conversor analógico digital para el registro de la señal transmitida a $40 \mathrm{kHz}$ por la NAU en Aguada Puerto Rico. Este equipo y una ubicación fuera de Tegucigalpa libre de interferencia, hicieron posible el registro de fulguraciones solares utilizando a la ionosfera.

Palabras Clave: VLF, Radio Definida a través de Software, Perturbaciones Súbitas en la lonósfera SID.

\section{Abstract}

In this document, we will discuss the correlation between solar flares and Sudden lonospheric Disturbances (SID), which were measure using the variability in the 
intensity of radio waves in the band of Very Low Frequency (VLF) reflected and refracted in the $D$ layer of the ionosphere and monitored outside of Tegucigalpa, in February of 2014.

With this in mind we build two distinct configurations which allow us to ensure quality in the data recollection phase. The first system is a UKRAA VLF receiver (United Kingdom Radioastronomy Association) with a bandwidth of 12 to $35 \mathrm{kHz}$ and an output in volts that represent the oscillations in the frequency of $24 \mathrm{kHz}$ NAA Cutler Maine. The last system takes advantage of Software Defined Radio (SDR) concepts which, without complex electronic hardware, analyze the VLF Signals using a modern sound card with an analog to digital converter for the data reception in 40.8 $\mathrm{kHz}$ transmitted by the NAU in Aguada Puerto Rico. This equipment and a facility in the suburbs of Tegucigalpa make possible the detection of solar flares using de ionosphere.

Keywords: VLF, Software defined radio SDR, Sudden lonospheric Disturbance SID

Adán Artola, (adanartola@hotmail.com) Edward Milla, (edward.milla@unah.edu.hn) Paolo Estrada (paolo.estrada.m@gmail.com) Facultad de Ciencias Espaciales / Departamento de Astronomía y Astrofísica Universidad Nacional Autónoma de Honduras (FACES I OACS - UNAH) Teléfono: 2239-4948 


\section{Introducción}

Desde sus inicios el OACS/UNAH contó con un telescopio óptico LX-200 de 16 pulgadas, que ha permitido realizar campañas de observación para el entrenamiento de los astrónomos de la Maestría en Astronomía y Astrofísica, con lo que se han logrado buenos resultados observacionales. Sin embargo, el observatorio está limitado a observaciones en el espectro visible y considerando que cielo de Tegucigalpa permanece nublado en buena parte del año, se ha pensado en aprovechar la parte no visible del espectro (radio) para aumentar la cantidad de observaciones, ya que estas depende muy poco de las condiciones climáticas.

Por tanto, este proyecto surge como una de las iniciativas del Departamento de Astronomía y Astrofísica de comenzar estudios en el área de radioastronomía, comenzando con equipo probado por organizaciones internacionales en el área de radioastronomía para el estudio Perturbaciones Súbitas en la lonosfera (SID).

Es conocido que la capa $D$ de la lonosfera es creada a través de la interacción entre flujos de energía en forma de Rayos X y Ultravioleta provenientes del Sol y elementos neutros como el Oxígeno y el Nitrógeno que se encuentran en la atmósfera de la Tierra, el flujo solar causa fotoionización o perdida electrones en los elementos neutros, provocando un aumento en la estructura electrónica de la capa $D$ y haciendo que esta se pueda utilizar como guía para las ondas de radio. El aumento en la concentración de electrones en la capa $D$ interactúa con las ondas de radio transmitidas desde tierra refractándolas de manera discreta hasta causar el reflejo de las mismas, permitiendo la comunicación a través de las bandas de Radio de Muy Baja (VLF) y Baja frecuencia (LF). Los cambios electrónicos anteriores también pueden originarse por Perturbaciones Súbitas en la lonósfera (SID), que son cambios en la estructura electrónica de la ionosfera causados por aumentos en la actividad solar o tormentas solares, que pueden provocar desde aumentos e interrupciones y hasta pérdidas completas de comunicación durante horas.

Las observaciones en la capa $\mathrm{D}$ de la ionosfera son escasas debido a que los satélites monitorean la atmósfera en altitudes superiores y los balones meteorológicos a alturas menores. Sin embargo las características principales de esta capa pueden ser estudias a través de las variaciones en la banda VLF de radio.

Para poder registrar estas perturbaciones en la lonósfera (SID) se utilizan receptores de frecuencias de radio de VLF y LF, estas bandas presentan pérdidas mínimas de potencia según su distancia recorrida las cuales pertenecen al orden de 2 a 3 decibelios $\mathrm{dB}$ cada $1,000 \mathrm{~km}$, gracias a esta característica es posible reci- 
bir señales de transmisores que se encuentran a miles de kilómetros de distancia, que operan a estas frecuencias de manera permanente, permitiendo un registro constante en tiempo de las variaciones de la señal de un porcentaje del globo y atmósfera terrestre.

Las fulguraciones solares son explosiones que ocurren en la superficie solar debido a la liberación de energía de los campos magnéticos solares generalmente sobre manchas solares. Las fulguraciones solares se clasifican según el brillo de estas en Rayos X subdividiéndolas según su intensidad como se logra observar en la Tabla 1. Las fulguraciones menos energéticas son de tipo $B$ y $C$, las de tipo medio se les clasifica $M$ y las más energéticas son de tipo $X$.

\begin{tabular}{|c|c|}
\hline Clase & $\begin{array}{c}\text { Intensidad en }\left(\boldsymbol{W} / \boldsymbol{m}^{2}\right) \text { en } \\
\text { longitudes de onda } \lambda \\
8 \leq \lambda \leq 9 \AA\end{array}$ \\
\hline $\mathrm{B}$ & $I<10^{-6}$ \\
\hline $\mathrm{C}$ & $10^{-6} \leq I<10^{-5}$ \\
\hline $\mathrm{M}$ & $10^{-5} \leq I<10^{-4}$ \\
\hline $\mathrm{X}$ & $I \geq 10^{-4}$ \\
\hline
\end{tabular}

Tabla 1. Clasificación de las Fulguraciones Solares según la NOAA

Los observatorios dedicados a las mediciones de la fluctuación energética solar son los Satélites Geoestacionarios Operacionales Ambientales (GOES como se conocerá desde ahora), el Satélite de Dinámica Solar (SDO como se conocerá desde ahora) que registran el flujo de Rayos-X (Longitudes de onda larga de 0.1 0.8 nanómetros, tomadas por el GOES 15) y Ultravioleta (Longitud de onda de 13.1 nanómetros tomada Ensamblador de Imágenes Atmosféricas (AIA) instrumento del $\mathrm{SDO}$ ) en ese orden. También existen otros observatorios que monitorean las variaciones VLF en diferentes partes del mundo, siendo los principales y que se utilizaran en este estudio los monitores de la Universidad de Stanford de ellos el monitor UTP 0389 ubicado en Pereira, capital del departamento de Risaralda en Colombia, coordenadas $\left(4.79^{\circ} \mathrm{N}, 75.69^{\circ}\right.$ O) y SMVA 0309 ubicado en el condado de Bedford, Estado de Carolina del Norte, EUA, coordenadas $\left(37.446^{\circ} \mathrm{N}, 79.523^{\circ} \mathrm{O}\right)$. 
Los monitores VLF utilizados en esta investigación registran las variaciones en la señal de dos transmisores principales el primero es la NAA ubicado en Cutler Maine, Estado de Maine, EUA, coordenadas $\left(44.644936^{\circ} \mathrm{N}, 67.281639^{\circ} \mathrm{O}\right)$ que transmite a la frecuencia de $24 \mathrm{KHz}$, el segundo transmisor la NAU de $40.8 \mathrm{KHz}$ ubicado en Aguada, Puerto Rico, coordenadas (18.398762 $\left.{ }^{\circ} \mathrm{N}, 67.177599^{\circ} \mathrm{O}\right)$.

En la investigación se buscó analizar las variaciones en la intensidad de la radiación electromagnética en Francisco Morazán como resultado de un evento Solar, tomando como base las variaciones en la señal de la banda de Muy Baja Frecuencia (VLF) de radio. Identificando la localización idónea de una base de monitoreo de eventos solares, seleccionado de un grupo de sitios alternativos en Tegucigalpa o alrededores que cumpla con los requisitos físicos y socioeconómicos. Obteniendo las variaciones en la intensidad de la señal en las bandas VLF de radio debido a los eventos solares, por medio de una unidad de monitoreo en radio. Y estableciendo si hay correlación entre los cambios en la intensidad de las señales de radio VLF y los fenómenos solares detectados en Francisco Morazán, con los datos registrados a nivel mundial.

\section{Metodología}

Para la realización del estudio se efectuaron las siguientes actividades:

\section{Caracterización del Sitio de Monitoreo}

Una causa importante de la interferencia en la banda VLF es el ruido de fondo, que en su mayoria es causado por emisoras de radio, lineas y centrales electricas. (Chernan, 1978). Es importante que el sistema de monitoreo se coloque alejada de fuentes de ruido a distancias promedio de (Department of the Navy Naval Electronic Systems Command, 1972):

- 400m de líneas de alta tensión

- $\quad 5 \mathrm{~km}$ de antenas de transmisión radio

- $8 \mathrm{~km}$ de comunidades e industrias

Para la captura de datos deben buscarse las mejores localizaciones para colocar el equipo. Para ello se definió de un grupo de tres lugares candidatos que presentaron características idóneas (poca interferencia radioeléctrica, disponibilidad de acceso a energía eléctrica, seguridad, etc.). 
Se eligió la comunidad de la Trinidad en el municipio de Sabana Grande a un promedio de 20.5 de kilómetros de distancia de la ciudad de Tegucigalpa en las coordenadas, $13.873547^{\circ} \mathrm{N}$, longitud $-87.248511 \mathrm{O}$. Ventajas físicas:

- Sitio alejado de comunidades e industria

- $\quad$ Nivel de interferencia bajo, lo demuestran mediciones realizadas en sitio.

\section{Diseño del Sistema para Monitoreo}

En la sección siguiente se detalla selección configuración de los receptores y antenas para el monitoreo dentro del proyecto:

- Receptor UKRAA Figura 1 (Asociación de Radioastronomía del Reino Unido): es un amplificador de banda estrecha de alta ganancia el cual se sintoniza a la frecuencia del transmisor NAA de $24 \mathrm{khz}$, las especificaciones del receptor se encuentra en la Tabla 2. El circuito que especifica la estructura del receptor se encuentra en la Figura 2. Este receptor utiliza un chip Maxim Max 186 para convertir la señal análoga a digital, estos datos son almacenados por el software Radio Skypipe y luego comparada con datos del satélite GOES 15 esto realizado por el software Starbase también por la UKRAA. El diagrama de la configuración del receptor y el equipo de cómputo se encuentra en la Figura3.

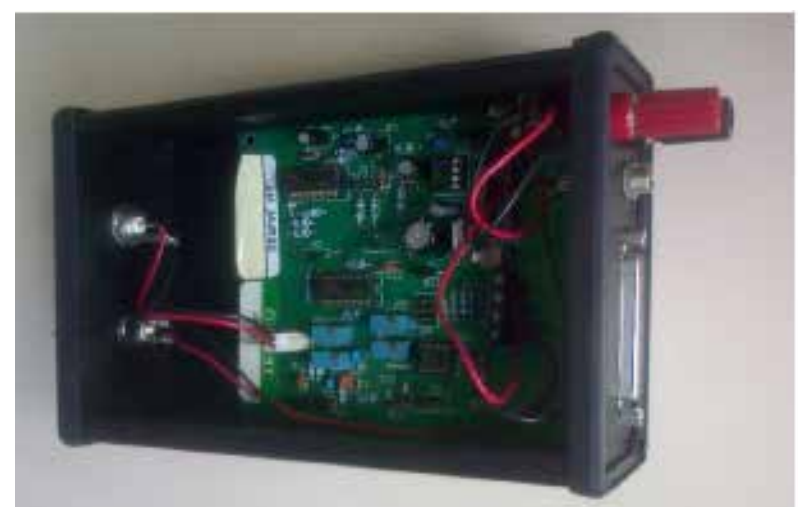

Figura 1. Receptor VLF UKRAA para la detección de Perturbaciones súbitas en la lonosfera. 


\begin{tabular}{|l|c|}
\hline \multicolumn{1}{|c|}{ Descripción } & Valor \\
\hline Rango de Sintonización & $12-35 \mathrm{kHz}$ \\
\hline Configuración de ganancia & $\mathrm{x} 1 \mathrm{y} \mathrm{x10}$ \\
\hline Salida de Voltaje & $0-5 \mathrm{~V}, 0-2.5 \mathrm{~V}$ \\
\hline Convertidor analógico/digital & Max 18612 bit \\
\hline Alimentación & $15 \mathrm{v}$ DC at 35mA \\
\hline
\end{tabular}

Tabla 2. Especificaciones del receptor VLF UKRAA

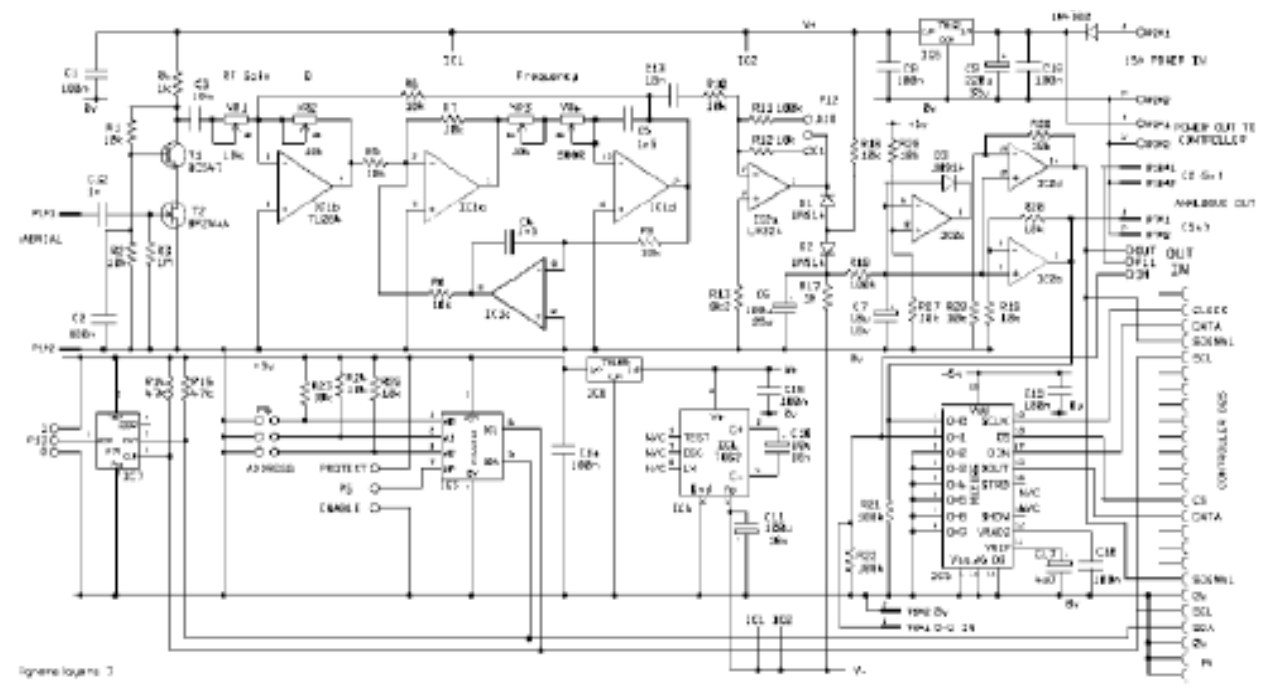

Figura 2. Diagrama de circuitos del receptor UKRAA 


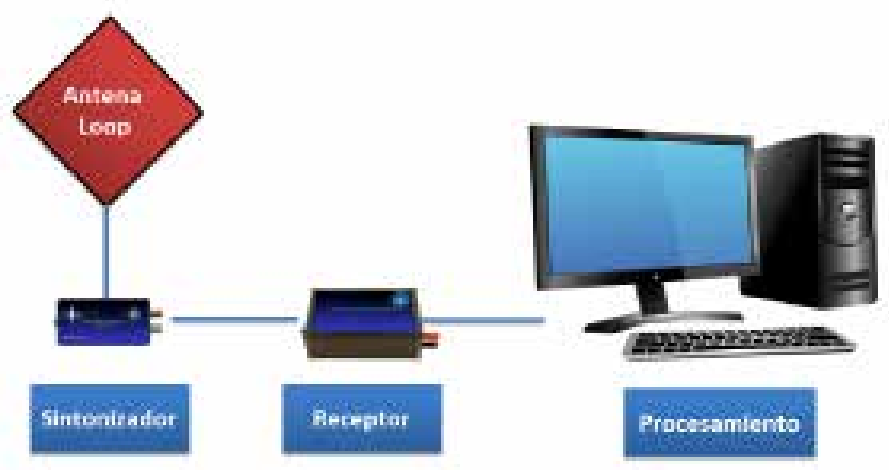

Figura 3. Diagrama de componentes del receptor VLF UKRAA

- Antena Octagonal: La antena octagonal Figura 4, fue utilizada para la recepción a través de los sistemas UKRAA, tiene 1 metro de Diámetro, la bobina consta de 127 vueltas de cable esmaltado calibre awg \#24. Esta Fue sintonizada para maximizar su ganancia a $24 \mathrm{KHz}$ Frecuencia del Transmisor VLF NAA situado en Cutler Maine.
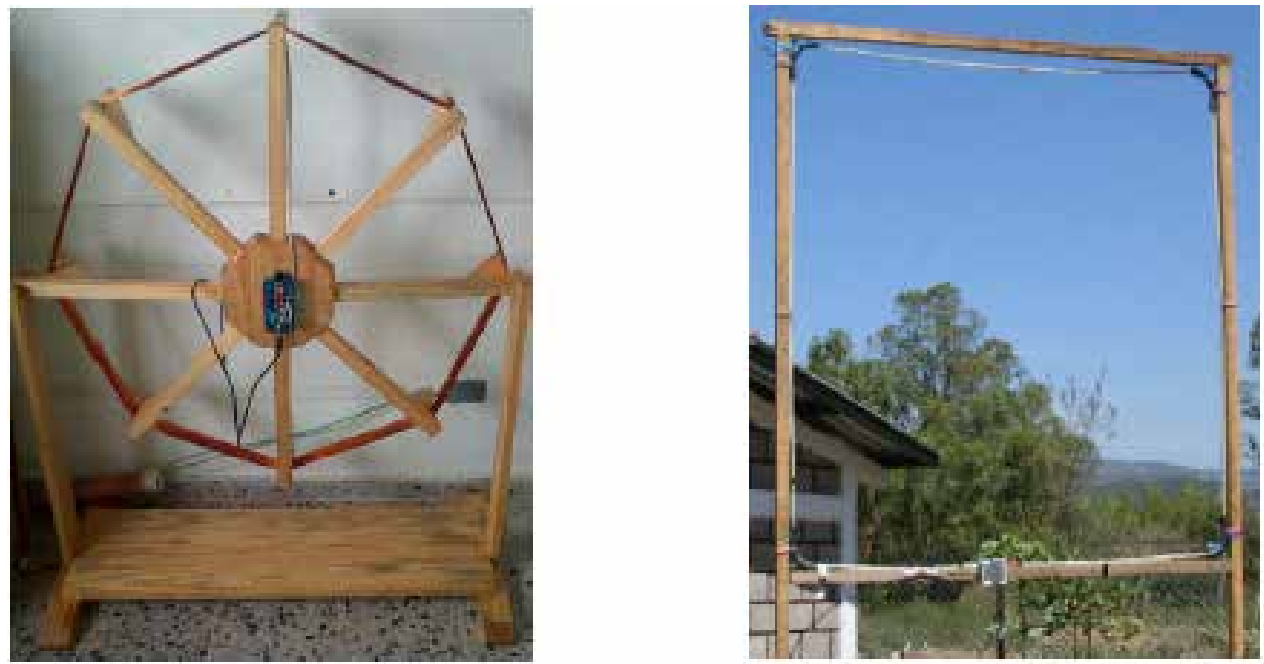

Figura. 4: a) Antena Loop conforma de octágono calibrada a $24000 \mathrm{Khz}$ frecuencia del transmisor NAA, Cutler Maine b) Antena Loop rectangular para software SDR 40.8 Khz frecuencia NAU Aguada Puerto Rico. 
- Receptor de SDR (Radio Definida a través de Software): los SDR son sistemas de radio que realizan el procesamiento de la señal a través de software en lugar de utilizar circuitos integrados en hardware.

El sistema SDR utiliza herramientas de informáticas de código libre para el monitoreo y análisis de las Perturbaciones Súbitas en la lonosfera SID. Se conforma por una antena loop cuadrada de madera de $2 m \times 2 m$ Figura 4 , en el cual se ajustó la bobina de $100 \mathrm{~m}$ repartidas en 12 vueltas de cable TFFT recubierto calibre awg \#18, esto para registrar la señal del transmisor NAU a $40.8 \mathrm{KHz}$. Se necesitó de un amplificador bajo en ruido que aumenta la ganancia en la señal, una tarjeta de sonido conectada al equipo de cómputo como convertidor analógico digital y a su vez para el procesamiento de la señal se utiliza la plataforma GNURadio, que sirve de base a la aplicación SIDSuite, encargada de filtrar la señal VLF y mostrar su comportamiento en forma gráfica. Las especificaciones de la tarjeta de sonido utilizada se muestran en la Tabla 3.

\begin{tabular}{|l|l|}
\hline \multicolumn{2}{|c|}{ Tarjeta de Sonido } \\
\hline \multicolumn{1}{|c|}{ Descripción } & \multicolumn{1}{c|}{ Valor } \\
\hline Frecuencia de respuesta & \multicolumn{1}{|c|}{$10-48 \mathrm{khz}$} \\
\hline Procesador & $\begin{array}{l}\text { AV100 High-Definition Sound } \\
\text { Processor (Max. 192KHz/24bit) }\end{array}$ \\
\hline $\begin{array}{l}\text { Rango de muestreo en } \\
\text { grabación y resolución }\end{array}$ & $\begin{array}{l}44.1 \mathrm{~K} / 48 \mathrm{~K} / 96 \mathrm{~K} / 192 \mathrm{KHz} @ \\
16 \mathrm{bit} / 24 \mathrm{bit}\end{array}$ \\
\hline
\end{tabular}

Tabla 3. Especificaciones Tarjeta de sonido

\section{Resultados}

- Se encontró un lugar que reúne las características para este tipo de estudio este lugar se ubica en la aldea la Trinidad del municipio de Sabana Grande, el cual cumple con parámetros establecidos para la recepción de la señal en las bandas de radio de Muy Baja Frecuencia (VLF) y Baja Frecuencia (LF).

- Diseño y construcción dos receptores de monitoreo SID, utilizando Receptor VLF de la UKRAA y un receptor de Radio definida a través de software (SDR) GNURadio y SIDSuite y Audio Spectrum Analyzer "Spectrum Lab" de los per- 
mitiendo de esta manera la redundancia y un mayor grado de confiabilidad en los datos obtenidos, además permite la evaluación de rendimiento entre los dos sistemas.

- Se ha realizado el diseño y construcción dos antenas para la recepción de datos en las bandas VLF y LF y la identificación de SID.

- Análisis de correlación en los datos.

Para el análisis de los datos se utilizó la gráfica de potencia de la señal VLF captada localmente versus las gráficas de Flujo de Rayos X y Ultravioleta captada por los satélites GOES y SDO en este orden, y también la señal VLF registrada po los monitores UTP 0383 y SMVA 0309 de la universidad de Stanford.

En la Figura 5 se muestra la potencia de la señal NAA 24kHz en decibelios, en este día no ocurrieron fulguraciones solares significativas, no se registraron perturbaciones, desde el inicio de la gráfica hasta el punto AM1 se encuentra la zona antes del amanecer en donde las ondas VLF son conducidas a través de la capa $E$ de la ionosfera esto hace que los cambios en la potencia de la señal sean impredecibles, la región entre los puntos AM1 y AM2 se encuentra la zona del amanecer local la cual es de transición y en donde comienza la formación dela capa $D$ de la ionosfera. La zona de día está comprendida entre los puntos AM2 y AT1, la formación de esta depende totalmente del ángulo del Sol al cenit. Dell punto AT1 hacia adelante se presenta la zona de transición del atardecer donde la.3 capa D desaparece nuevamente. Estas transiciones son repetitivas para los días sin perturbaciones . (More, Sharma, Bhonsle, \& Lynn, 2010).

El cruce datos entre fulguraciones y perturbaciones VLF se dio en los eventos que tuvieron lugar a la misma hora como se representa claramente en la Figura 6, En esta se logra observar el comportamiento de la señal VLF durante un aumento en el flujo de energía del Sol, esto gracias a una fulguración de la clase C e intensidad 7.7 registrada por los satélites GOES y SDO y el monitor VLF UTP 0383. 


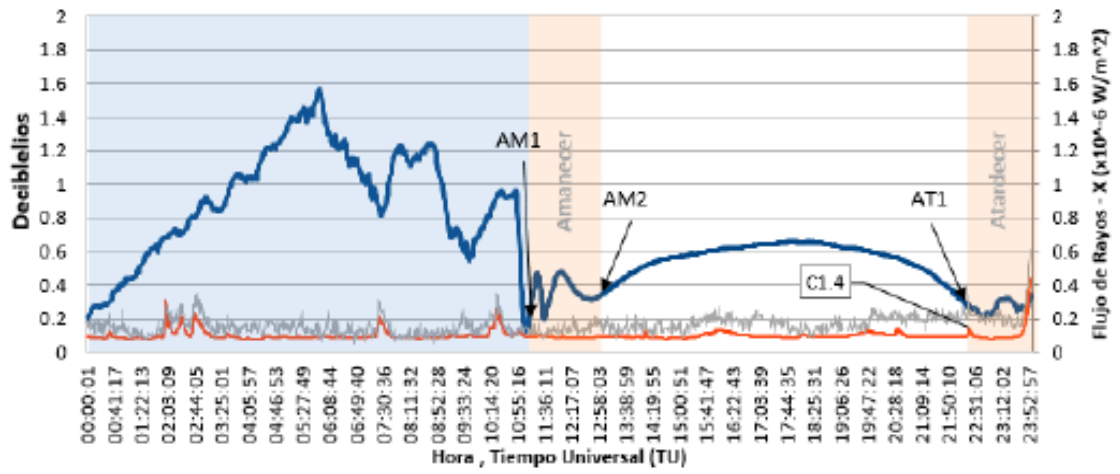

Figura. 5: Se presenta la gráfica de 24horas de la señal Local NAA 24KHz (Línea Azul), el flujo en Rayos- $X$ registrado por los Satélites GOES (Línea roja), En gris la intensidad de flujo de rayos ultravioleta (UV) tomada por el satélite SDO. Las variaciones 10:55TU (Tiempo universal) a 13:00 TU, y de 22:10 TU a 23:13 TU, son las fases inicial y final de recombinación de la ionósfera por lo que no se toman en cuenta.

\section{Local NAA 24KHz vs GOES, SDO,UTP - TU 22/02/2014}
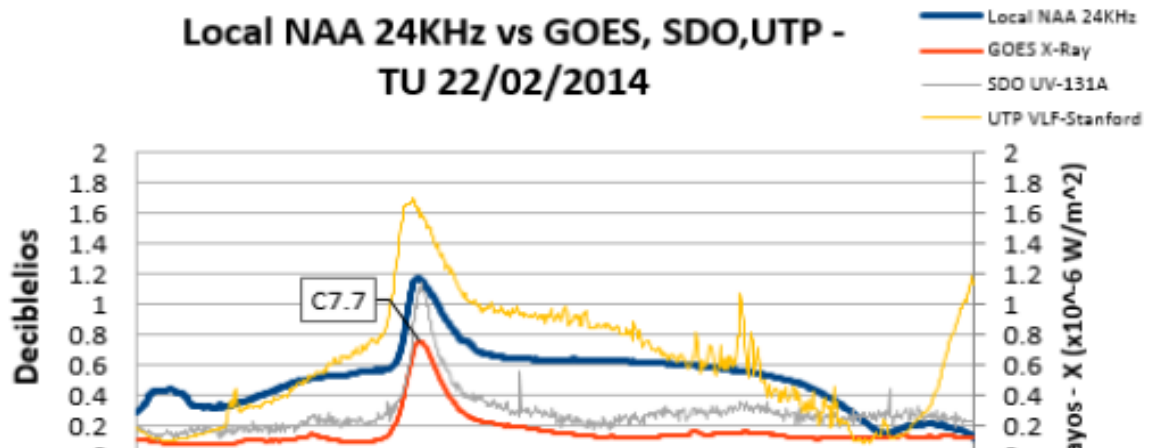

0

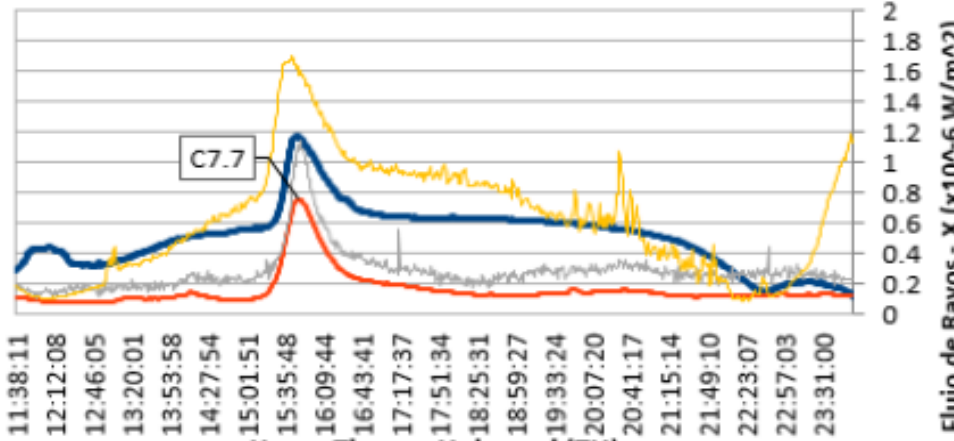

Hora, Tiempo Universal (TU)

Figura. 6: En esta figura se presenta la gráfica de día de la señal Local NAA 24KHz (Línea Azul), la cual se ve afectada por una fulguración de intensidad C7.7 detectado en Rayos-X por los Satélites GOES (Línea roja). En gris se presenta la intensidad de flujo de rayos ultravioleta (UV) tomada por el satélite SDO y la línea naranja representa las variaciones VLF detectadas por el receptor UTP 0383 de la Universidad de Stanford. Las variaciones 11:38 TU (Tiempo universal) a 12:03 TU, y de 22:27 TU a 23:59 TU, son las fases inicial y final de recombinación de la ionósfera por lo que no se toman en cuenta. 
Para los efectos de determinar cuál es el cambio en la potencia de la señal VLF se muestra la Figura 7 en donde se realiza la comparación entre el día 22 de febrero (Día con perturbación) y el día 19 de febrero de 2014 (Día sin perturbación), lo cual nos permite observar con mayor claridad el cambio súbito de potencia con respecto a un día sin perturbación.

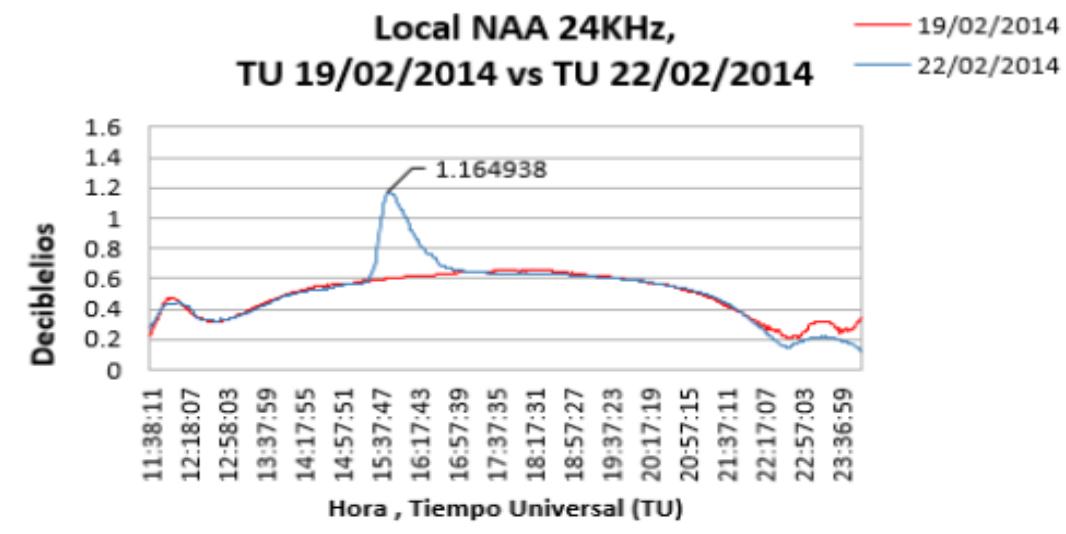

Figura. 7: Este grafico se presenta la comparación entre las potencias de la señal del transmisor NAA el día 19/02/2014 sin perturbaciones (línea roja) y el día 22/02/2014 con perturbaciones (línea azul), esto para resaltar las variaciones de potencia que se dan durante los eventos solares.

Para comparación entre los días con múltiples perturbaciones se tomaran los días 14 y 15 de febrero de 2014 en la Figura 8 donde se pudieron detectar cerca de 4 fulguraciones de las 6 fulguraciones que tuvieron lugar ese día, lo que nos permite verificar como la potencia de la señal VLF varía según los cambios de intensidad del flujo de rayos $\mathrm{X}$ que inciden en la ionosfera con el tiempo. 


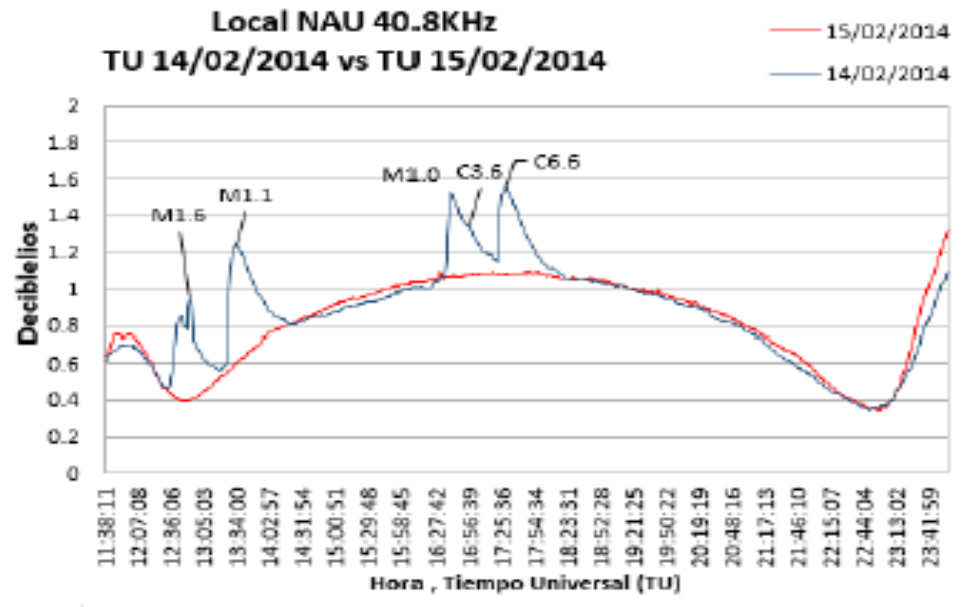

Figura 8: Este grafico se presenta la comparación entre las potencias de la señal del transmisor NAA el día 15/02/2014 sin perturbaciones (línea roja) y el día 14/02/2014 con perturbaciones (línea azul), lo que muestra los diferentes tipos de fulguraciones que pueden afectar la señal.

\section{Discusión}

- La aldea la Trinidad en el municipio de Sabana Grande, se encuentra a 20.5 $\mathrm{km}$ de la ciudad de Tegucigalpa, las fuentes de interferencia más próximas se encuentran a $5.24 \mathrm{~km}$. además de ser una comunidad aislada con actividad radioeléctrica mínima. Todo esto garantiza condiciones aceptables al presente estudio.

- Se optó por la configuración de hardware y software, descrita en este documento debido a sus costos bajos, flexibilidad en la configuración, robustez y capacidad de procesamiento aceptable para el propósito presente estudio.

- Los datos obtenidos con el equipo local demuestran una correlación con los eventos solares registrados por otros observatorios, pero aún se necesita un periodo de tiempo de observación más prolongado para determinar con precisión el nivel de esta correlación.

\section{Conclusiones}

- Debido a la revisión bibliográfica de estándares, a su anterior uso como sitio de monitoreo de señales de Muy Baja Frecuencia (VLF) y Baja Frecuencia (LF) 
y la visita al sitio se concluye que la aldea la Trinidad es un lugar apto para la recepción de señales y detección de perturbaciones en la ionosfera.

- Es posible construir equipo de bajo costo para el monitoreo de la actividad solar, para nuestra posición geográfica y niveles de ruido aceptables para el estudio propuesto en este artículo como en el caso del tamaño de las antenas, pero que permiten la recepción, análisis y almacenamiento de las ondas de la banda VLF y por lo tanto el registro de Perturbaciones Súbitas en la lonosfera (SID).

- Gracias a las observaciones realizadas diariamente a las señales VLF del NAA $24 \mathrm{KHz}$ y NAU $48 \mathrm{KHz}$ y al comprobar que el número de variaciones captadas de manera local con el equipo VLF tienen la misma frecuencia que los eventos de fulguraciones registrados por el GOES, se puede concluir que para el tiempo de observación con el Equipo VLF existe correlación entre las Perturbaciones Súbitas en la lonosfera con el variaciones de potencia VLF registradas con el equipo local.

\section{Recomendaciones}

A la hora de realizar estudios VLF de alta sensibilidad será necesario realizar estudios de piso ruido e interferencias que nos permitan conocer cuáles son las condiciones a las cuales se encuentra el sitio de monitoreo, unido a esto también será un gran aporte a la investigación que las instalaciones del sitio de monitoreo tengan acceso a internet lo que permitirá un monitoreo remoto continuo de la información y del estado equipo, también es importante el uso de baterías que aseguren un flujo de energía constante.

En esta investigación se utilizó dos equipos de radio UKRAA y Radio Definida a través de Software, el equipo SDR la cual se recomienda ya que permite acceder a todos los canales de la banda VLF para mantener el registro continuo de varios transmisores, también tiene un costo, un tiempo y complejidad de construcción, menor, por lo que es la primera alternativa para la recepción de SID.

Es necesario realizar análisis de las SID durante periodos prolongados de tiempo lo que permitirá registrar cambios estacionales de la señal VLF recibida y realizar nuevas correlaciones entre datos, también esto ayudara a establecer los límites en nuestras mediciones y llevar un registro continuo de la actividad solar en la ionosfera. 


\section{Bibliografía}

- American Association of Variable Star Observers (AAVSO). (2012, Junio 14). Sudden lonispheric Disturbances (SIDs). Retrieved 11 08, 2012, from American Association of Variable Star Observers (AAVSO): http://www.aavso.org/ solar-sids

- Haslett, C. (2008). Essentials of Radio Wave Propagation. Ofcom, UK: Cambridge University Press.

- Richards, J. A. (2008). Radio Wave Propagation An Introduction for the Non-Specialist. Canberra, Australia: Springer.

- Aeropuertos de Honduras. (2013). Reporte estadístico mensual. Tegucigalpa: No publicado.

- Aeropuertos de Honduras SA. (2012). Manual de procedimiento de cobro por servicios aeroportuarios. Tegucigalpa, Francisco Morazán , Honduras: No publicado.

- Alberto Colindres, A. M. (2014). Cuerpo de Contenido para la Formación de Controlador de Tránsito Aéreo en el ámbito de las ciencias aeronáuticas en Honduras. Tegucigalpa: No publicado.

- American Association of Variable Star Observers (AAVSO). (2012, Junio 14). Sudden lonispheric Disturbances (SIDs). Retrieved 11 08, 2012, from American Association of Variable Star Observers (AAVSO): http://www.aavso.org/ solar-sids

- American Meteorological Society. (2012). Glossary of meteorology. Allen Press.

- Castillo, Y. (2012). Diseño de un radiotelescopio para actividades de investigación . Revista de Ciencias espaciales, --.

- Chernan, C. (1978). The handbook of solar flare monitoring \& propagation forecasting. Blue rdge, USA: Tab Books. 
- Committee on the Societal and Economic Impacts of Severe Space Weather Events. (2008). Severe Space Weather Events - Understanding Societal and Events: A Workshop. Washington, DC, United States of America: The National academy Press.

- Cummer, S., Inan, U., \& Bell, T. (1998, Noviembre-Diciembre). lonospheric D region remote sensing using VLF radio atmpspherics. Radio Science, 33(6), 1781-1792.

- Department of the Navy Naval Electronic Systems Command. (1972). Naval Shore Electronic Criteria: VLF, LF, and MF Comunication Systems. Washington: U.S. Printing Office.

- DGAC. (2004). Ley de Aeronáutica Civil. Tegucigalpa: DGAC.

- DGAC Chile. (2012, Noviembre 22). DGAC. Retrieved from www.dgac.gob.cl/ portal

- Dixon, B. (1991). " La Arquitectura del periodo Formativo y la competencia del estado social en Yarumela, Honduras. In YAXKIN. Honduras .

- Dixon, B. (2008). Yarumela: una historia de investigacion arqueologica en el sitio y su lugar en la antigua historia hondureña. In R. YAXKIN, Revista del instituto hondureño de antropologia e historia (Vol. XXIV, p. 199). Tegucigalpa, Honduras: ENAG.

- Embry Riddle. (2012). Undergraduate/Graduate Catalog. Daytona: Embry Riddle.

- FUMEC. (2012, Noviembre 27). Universidade FUMEC. Retrieved from www. fumec.br/cursos

- Grubor, D., Suli, D., \& Zigman, V. (2005). Influence Of Solar X-Ray Flares On The Earth-lonosphere Waveguide. Serbian Astronomical Jounal, 29-35.

- Haridas, D., Soman, K. P., \& Sundaram, S. (2013). Ionospheric Disturbances due to Solar lonospheric Disturbances due to Solar. International Journal of Advanced Electrical and Electronics Engineering, 69-74. 
- Hunsucker, R. D., \& Hargreaves, J. K. (2003). The high-latitude ionosphere and its effects on radio propagation. Cambridge, UK: Cambridge University Press.

- Interairport, Estado de Honduras. (2000, septiembre 23). Contrato de concesiones. Tegucigalpa, Honduras: La Gaceta.

- J.S. Perez, E. M. (1999). Manual Sanitario para Tripulantes de Cabina de Pasajeros. Madrid: Arán Editores.

- Kolarski, A., Grubor, D., \& Suli, D. (2011, Agosto 8). Diagnostics Of The Solar $X$-Flare Impact On Lower lonosphere Through The VIf-Naa Signal Recordings. Baltic Astronomy, 20, 591-595.

- Lang, J.-P. (2013, 05 10). GNU Radio. Retrieved from Welcome to GNU Radio!: http://gnuradio.org/redmine/projects/gnuradio/wiki

- Lashley, J. (2010). The Radio Sky and How to Observe It. (M. Inglis, Ed.) New York, USA: Springer.

- Leech, M. (2014, 03 28). Proyecto SIDSuite. Retrieved from The Comprehensive GNU Radio Archive Network: https://www.cgran.org/browser/projects/SIDSuite

- Mandeville, J. (1986). "Proyecto Arqueologico Valle de Comayagua: Investigaciones en Yarumela-Chical. IX N² 2,1986, 17-41.

- Mandeville, J. (1997). "Programa Arqueologico de Yarumela, temporada 1983: descubrimientos y analisis". XV, edicion extraordinaria 1990-1994, 5-18.

- More, C., Sharma, A., Bhonsle, R., \& Lynn, K. (2010). Fiel Strength Measurement of VLF Waveguide of VLF RadioWave Propagation at $19.8 \mathrm{KHz}$ betwen Australia and India. Australian Space Science Conferene Series (pp. 249-262). Sydney: Wain.

- Nakamura, S. (1991). Desarrrollo y decaimiento en la periferia de Copán". annals of Latin American studies, 39-95.

- National Bureau of Standards. (1937). Radio Instruments and Measurements. Washington: GOVERNMENT PRINTING OFFICE. 
- NOAA/Space Weather Prediction Center. (2012, Octubre 8). Space Weather Prediction Center. Retrieved Octubre 27, 2012, from http://www.swpc.noaa. gov/SolarCycle/index.html

- OACI. (2006). Doc 9562 Manual sobre los aspectos económicos de los aeropuertos, Segunda Edición. Montreal: $\mathrm{OACl}$.

- OACI. (2011). Estudio de la OACI revela gran demanda de personal aeronáutico cualificado de aquí al Año 2030. OACl.

- OACI. (2012, Noviembre 20). ICAO. Retrieved from icai.int/safety/dangerous good radiosky.com. (2013, 10 30). Radio-SkyPipe II - An Internet Enabled Strip Chart Recorder. Retrieved from Radio-SkyPipe II - An Internet Enabled Strip Chart Recorder: http://www.radiosky.com/skypipeishere.html

- Raulin, J., Berton, F., Gavilán, H., Guevara, W., Rodriguez, R., Fernandez, G., . .. Hadano, R. (2010). Solar flare detection sensitivity using the South America VLF(SAVNET). Journal Of Geophysical Research, 115, A07301.

- Raulin, J., Correia de Matos, P., Hadano, R., Saraiva, A., Correia, E., \& Kaufmann, P. (2009). The Souh America VLF Network (SAVNET):Development installation status, first results. Geofísca Internacional, 48(3), 253-261.

- Sampieri, H. (2010). Metodología de la investigación. México: McGraw Hill.

- spaceweather.com. (n.d.). The Classification of X-ray Solar Flares. Retrieved Octubre de 2010 28, from http://spaceweather.com: http:/spaceweather.com/ glossary/flareclasses.html

- U S P M. (2012, Noviembre 27). Universidad San Martín de Porres. Retrieved from www.usmp.edu.pe/ffia/aeronauticas

- UKRAA. (2013, 10 30). UK Radio Astronomy Association. Retrieved from UK Radio Astronomy Association: http://www.ukraa.com/www/

- Universidad Técnica Federico de Santa María. (2012, Noviembre 27). Ingeniería en aviación comercial. Retrieved from www.usm.edu.cl/admision

- USAC. (n.d.). Atlas Arqueologico de Guatemala. (D. G. Natural, Producer) Retrieved from http://www.atlasarqueologico.com/. 
- Valladarez, O. A. (2008, Septiembre). Yarumela: Revalorizacion un sitio aqueológico en el valle de Comayagua. (ENAG, Ed.) YAXKIN, XX1V, No1, 211-2226.

- Vivanco, M. (2005). Muestreo estadístico, diseño y aplicaciones. Santiago.

- Wolf, D. (2013, 10 30). Audio Spectrum Analyzer . Retrieved from Audio Spectrum Analyzer : http://www.qsl.net/dl4yhf/spectra1.html 